\title{
Alimentación en gestantes adolescentes con hipertensión: Cuidados y Cultura
}

\author{
Diet in pregnant teenagers with hypertension: Care and Culture
}

Wendy Johana Gómez-Domínguez ${ }^{*}$ orcid.org/0000-0001-5101-0741

1. Programa de Enfermería, Universidad de Sucre. Sincelejo, Colombia

Fecha de recepción: Febrero 08 - 2021

Fecha de revisión: Agosto 23 - 2021

Fecha de aceptación: Diciembre 20 - 2021

Gómez-Domínguez WJ. Alimentación en gestantes adolescentes con hipertensión: Cuidados y Cultura. Univ. Salud. 2022;24(1):29-35. D0I: https://doi.org/10.22267/rus.222401.254

\section{Resumen}

Introducción: La gestación en adolescentes es una realidad que enfrentan cada día la familia, comunidad y sociedad; además puede tener complicaciones como la hipertensión, por lo tanto, los cuidados son fundamentales y pueden variar según la cultura. Objetivo: Describir las prácticas de cuidados culturales relacionadas con la alimentación de las gestantes adolescentes con hipertensión, hospitalizadas en una institución de III Nivel de Atención en Salud del municipio de Sincelejo. Materiales y Métodos: Investigación cualitativa etnográfica. Se aplicaron entrevistas semiestructuradas a profundidad a trece participantes. El análisis etnográfico permitió identificar términos, dominios, taxonomías y relaciones semánticas. Resultados: Se observó un alto consumo de alimentos tradicionales de la región Caribe y productos procesados; emergieron prácticas de cuidados referentes a los cambios en la alimentación y su significado cultural, en las subcategorías, alimentos saludables y alimentos relacionados con hipertensión. Conclusiones: Las prácticas de cuidados en la alimentación, evidencian la diversidad de significado cultural en las participantes, conocimiento esencial para brindar cuidados de enfermería coherentes y humanizados. Asimismo, conlleva a la implementación de programas de intervención a nivel domiciliario e institucional en esta población.

Palabras clave: Prácticas culturales; cuidados de enfermería; alimentación; gestación en adolescencia. (Fuente: DeCS, Bireme).

\begin{abstract}
Introduction: Pregnancy in adolescents is a common reality faced by family, community and society, which can also bring health complications such as hypertension. Therefore, care of these teenagers is fundamental and can vary according to cultural contexts. Objective: To describe cultural care practices related to diet in pregnant adolescents with hypertension, who are inpatients in a Level III health care institution in Sincelejo (Colombia). Materials and methods: Qualitative ethnographic research. In-depth semistructured interviews were done with thirteen participants. The ethnographic assessment facilitated the identification of terms, domains, taxonomies, and semantic relationships. Results: High consumption of traditional Caribbean food and processed products was observed. Care practices related to diet changes and their cultural meaning were common in subcategories such as healthy food and hypertension related diet. Conclusions: Diet care practices show diversity in terms of the cultural significance given by participants, which is an essential knowledge to provide coherent and humanized nursing care. Likewise, it is necessary to implement intervention programs to treat these patients both at home as well as at health care institutions.
\end{abstract}

Keywords: Cultural characteristics; nursing care; diet; pregnancy in adolescence. (Source: DeCS, Bireme).

\footnotetext{
*Autor de correspondencia

Wendy Johana Gómez Domínguez

e-mail: Wendy.@unisucrevirtual.edu.co
} 


\section{Introducción}

La reproducción del ser humano, es una necesidad y a la vez una meta en el camino de la existencia, pero es en esa vía donde la gestación se convierte en un estado fisiológico deseado por muchas familias e indeseado por otras, debido a que son muchos los factores que intervienen en este período de la vida, existiendo factores individuales, familiares, socioeconómicos, culturales y psicológicos, los cuales convierten la gestación en una etapa de riesgo(1-3).

La gestación en la adolescencia es considerada un problema de salud pública y a la vez uno de los principales factores que favorecen a la mortalidad materna e infantil y al círculo de enfermedad y pobreza. A inicios de 2020, la Organización Mundial de la Salud (OMS) reportó "16 millones de gestantes adolescentes entre 15 a 19 años y alrededor de 1 millón de menores de 15 años dando a luz cada año", donde la mayoría habita en países de ingresos bajos y medios. Además, los recién nacidos de madres adolescentes tienen una mayor probabilidad de registrar bajo peso al nacer y prematuridad(4).

En Colombia, según el boletín epidemiológico semanal del Instituto Nacional de Salud para la semana epidemiológica 53 del año 2020 se notificaron 577 muertes maternas, de ellas 409 tempranas (ocurridas durante el embarazo, parto y hasta los 42 días de terminada la gestación). Dentro de las principales causas de muerte se encontró la hemorragia obstétrica con el $15,9 \%$ y el trastorno hipertensivo asociado a la gestación con 13,7\%, seguido por neumonía por coronavirus (COVID-19) con el 12,0\%; cifras que incitan a trabajar en equipo desde la profesión de enfermería(5).

El departamento de Sucre ubicado en la región Caribe de Colombia, está conformado por las subregiones: Golfo de Morrosquillo, Montes de María, Sabanas, Mojana y San Jorge; según la Secretaría Departamental de Salud en el año 2016, reportó 3.068 gestantes adolescentes; específicamente en Sincelejo, capital del departamento de Sucre, perteneciente a la subregión Sabanas, se mantuvo con el mayor número de casos (991 casos) (6). No se encontraron registros de investigaciones relacionadas con las prácticas de cuidado cultural en adolescentes embarazadas y una institución de III Nivel de Atención en Salud en el municipio de Sincelejo fue la que reportó casos significativos de adolescentes con hipertensión inducida por la gestación(7).
Desde el anterior contexto y con la certeza de que la disciplina de enfermería siempre ha estado presente en el cuidado de la salud, como pieza clave en el pasado, presente y futuro de la humanidad, fue imprescindible llevar a cabo esta investigación con el objetivo de describir las prácticas de cuidados culturales relacionadas con la alimentación de las gestantes adolescentes con hipertensión, hospitalizadas en una institución de III Nivel de Atención en Salud del municipio de Sincelejo. Este estudio permitirá posicionar la atención integral nutriendo la capacidad resolutiva de enfermería al valorar en el entorno de cada adolescente los cuidados culturales, igualmente, contribuirá al fortalecimiento de intervenciones en la institución de salud y de la Secretaría Departamental de Salud de Sucre en este grupo poblacional.

\section{Materiales y métodos}

\section{Tipo de estudio}

Investigación cualitativa etnográfica, enfoque de etno-enfermería, de la teorista Madeleine Leininger, destacada como la pionera de la Enfermería Transcultural, indica que el cuidado tiene semejanzas y diferencias dependiendo del entorno cultural, y es donde enfermería actúa como puente de unión entre el sistema genérico y el profesional, estableciéndose tres tipos de cuidados enfermeros, mantenimiento, negociación y reestructuración de los cuidados culturales, para brindar cuidado coherente con la cultura(8-10). Estudio efectuado en el período comprendido del mes de diciembre de 2017 hasta abril de 2018, logrando la saturación teórica; participaron 13 personas, 9 como informantes clave: Adolescentes con hipertensión inducida por la gestación y 4 personas como informantes generales: madres, abuelas, o cuñadas. Las adolescentes se encontraban en los servicios de Sala de Partos, Hospitalización General, Unidad de Cuidados Intermedios y Unidad de Cuidados Intensivos, de una institución de III Nivel de Atención en Salud del municipio de Sincelejo.

\section{Criterios de inclusión y exclusión}

Los criterios de inclusión fueron: Adolescentes con hipertensión inducida por la gestación (conocido también como hipertensión gestacional), entre los 14 y 19 años de edad, quienes expresaron y firmaron el consentimiento para participar en el estudio y no cursar con diagnóstico de otra patología. Madres, abuelas, suegras, cuñadas y hermanas, que convivían con la adolescente, que actuaron como informantes clave. Se excluyeron las gestantes adolescentes que padecían problemas de salud mental, las menores de 
14 años y mayores de 19 años, y madres, abuelas, suegras, cuñadas o hermanas, que no convivían con las adolescentes.

\section{Muestra}

La población total fue de 19 personas y el muestreo se determinó por pertinencia, adecuación, conveniencia, oportunidad y disponibilidad, con la participación de 13 personas, 9 informantes clave y 4 informantes generales. Las adolescentes fueron captadas en los servicios de Sala de Partos (5), Hospitalización General (2), Unidad de Cuidados Intermedios (1) y Unidad de Cuidados Intensivos (1), a través de una matriz de ingresos y egresos, en este lugar se acordó una cita para la primera entrevista y también se realizaron llamadas telefónicas. Se construyó una base de datos en Excel que permitió la organización y ubicación de las gestantes.

\section{Recolección y Análisis de la información}

Se realizó la inmersión al campo y luego la recolección de la información a través de entrevistas etnográficas y la observación participante(11), esto permitió que las personas proporcionaran la información sobre sus vidas, relacionada con el fenómeno de estudio.

Después de ejecutadas las entrevistas en las viviendas (tiempo promedio de 45 minutos), observación participante, notas de campo y fotografías, se realizó la transcripción de cada una (antes de las 72 horas), mediante un código asignado a la entrevista y otro código asignado a la información, con una duración en la transcripción de 3 a 5 horas, posteriormente se hizo el análisis según lo propuesto por la teorista Leininger(8-10) (identificando descriptores, realizando la categorización, identificación de patrones de valores, creencias, prácticas, entorno e identificación de categorías y subcategorías) que posibilitó el análisis etnográfico identificando términos incluidos, relaciones semánticas, dominios y taxonomías. Una vez se realizaba la primera entrevista, se analizaba, se preparaba la siguiente entrevista y se guardaba en una memoria USB y Grabadora periodística.

\section{Consideraciones éticas}

El estudio fue avalado por la institución de III nivel de Atención en Salud, el Comité de Ética de la Facultad de Enfermería de la Universidad Nacional de Colombia y el consentimiento de las participantes; cumpliendo con lo contemplado en la Resolución 008430, del 4 de octubre de 1993(12), normatividad vigente de la investigación en salud en Colombia; clasificando según el numeral b del Artículo 11, en "investigación sin riesgo". Se enmarcó dentro de los principios éticos contemplados en el artículo 2 de la Ley 911/2004: No maleficencia, justicia, beneficencia, autonomía, confiabilidad, veracidad y fidelidad(13).

\section{Resultados}

Se visitó cada una de las viviendas de las informantes, ubicadas en el municipio de Sincelejo en los sectores: Botero, Villa Katy, San Remo, Sevilla, Villa Orieta, Cielo azul, y Ciudad Jardín, zonas donde se presentan muchas las dificultades para cubrir los servicios básicos del día a día; las viviendas están clasificadas en estrato socioeconómico uno, el acceso a cada vivienda fue difícil ya que en estas áreas están presentes el peligro y la inseguridad social. En cuanto al grado de escolaridad de las participantes, predominó la secundaria incompleta en un 77,8\%.

El entorno cultural contribuyó a profundizar sobre la vida de la gestante adolescente y su núcleo familiar. En lo referente a la alimentación es una zona en la que predominan alimentos característicos de la región Caribe, tales como, arroz, maíz, yuca, ñame, plátano, hortalizas, frutas tropicales (naranja, mandarina, limón, guayaba, patilla, nísperos, melón, maracuyá, piña), pescados, carne bovina, porcina, de aves de corral (gallinas, pavos), lácteos y productos procesados.

La investigación evidenció las prácticas de cuidados en la alimentación y su significado cultural correspondientes a la categoría: Cambios en la alimentación de la gestante adolescente. Estos se subdividieron en las subcategorías: Alimentos saludables y Alimentos relacionados con desarrollo de hipertensión arterial; donde se identificaron: 8 dominios y 12 taxonomías (Tabla 1). Se originaron las relaciones semánticas: de inclusión y de causa efecto.

Existe gran variedad de prácticas de cuidados culturales en las gestantes adolescentes participantes, quienes se encontraban en un entorno donde los aspectos socioculturales y económicos influyeron en la alimentación de cada adolescente y su núcleo familiar.

Los hallazgos de este estudio, muestran que la variedad de alimentos catalogados como saludables es significativa, lo que representa una relación semántica de inclusión (Tabla 2). En esta subcategoría, el plátano se constituye en un alimento que en el entorno físico de las gestantes es fácil de obtener en las carretas campesinas que se ubican en la puerta de los domicilios y se adquiere a un bajo precio. 
Tabla 1. Prácticas de Cuidado Cultural en la alimentación de las gestantes adolescentes con hipertensión arterial. Sincelejo, 2017 - 2018

\begin{tabular}{|c|c|c|c|}
\hline Categoría & Subcategorías & Dominios & Taxonomías \\
\hline \multirow{13}{*}{$\begin{array}{l}\text { Cambios en la } \\
\text { alimentación de la } \\
\text { gestante } \\
\text { adolescente }\end{array}$} & \multirow{11}{*}{$\begin{array}{l}\text { Subcategoría } 1 . \\
\text { Alimentos saludables. }\end{array}$} & $\begin{array}{l}\text { Dominio 1. Comida para evitar las } \\
\text { náuseas en la gestación. }\end{array}$ & \multirow{8}{*}{$\begin{array}{l}\text { Taxonomía 1. Propiedades de los } \\
\text { alimentos tolerados en la gestación. } \\
\text { Taxonomía 1. Carnes magras, pollo y } \\
\text { pescado. } \\
\text { Taxonomía 2. Ñame criollo y plátano } \\
\text { verde } \\
\text { Taxonomía 3. La espinaca. } \\
\text { Taxonomía 4. Sopas de lentejas y de } \\
\text { frijoles rojos. } \\
\text { Taxonomía 1. Mandarinas } \\
\text { Taxonomía 2. La granadilla sirve para } \\
\text { todo } \\
\text { Taxonomía 3. Propiedades del extracto } \\
\text { de remolacha } \\
\text { Taxonomía } 4 \text {. Infusión de manzanilla y } \\
\text { anís en la noche }\end{array}$} \\
\hline & & Dominio 2. Consumo de dieta saludable. & \\
\hline & & & \\
\hline & & & \\
\hline & & Dominio 3. Meriendas saludables. & \\
\hline & & & \\
\hline & & & \\
\hline & & & \\
\hline & & $\begin{array}{l}\text { Dominio 4. Comer a tiempo para } \\
\text { disminuir el reflujo y sobrepeso en la } \\
\text { gestación. }\end{array}$ & \\
\hline & & Dominio 5. Consumir agua en la noche. & Taxonomía 1. Agua hervida \\
\hline & & $\begin{array}{l}\text { Dominio } 6 . \text { Satisfacción y momentos } \\
\text { felices para la gestante. }\end{array}$ & \\
\hline & \multirow{2}{*}{$\begin{array}{l}\text { Subcategoría } 2 . \\
\text { Alimentos } \\
\text { relacionados con } \\
\text { desarrollo de } \\
\text { hipertensión arterial. }\end{array}$} & $\begin{array}{l}\text { Dominio 1. Ingesta de dulces (Arequipe, } \\
\text { bocadillo, chocolate). }\end{array}$ & \multirow{2}{*}{$\begin{array}{l}\text { Taxonomía 1. Los panes causan } \\
\text { sobrepeso } \\
\text { Taxonomía 1. Arroz tostado (pegao o } \\
\text { cucayo del arroz). }\end{array}$} \\
\hline & & $\begin{array}{l}\text { Dominio 2. Hipertensión arterial en la } \\
\text { gestante adolescente (Consumo de } \\
\text { alimentos influyentes). }\end{array}$ & \\
\hline
\end{tabular}

En la dieta de las gestantes también se presentan alimentos que están relacionados con el desarrollo de la hipertensión arterial, lo que indica una relación semántica: causa - efecto (Tabla 3). Alimentos que hacen parte de la cocina de las participantes, los cuales no proporcionan beneficios para la salud de las gestantes adolescentes y de los miembros de la familia.

Tabla 2. Alimentos saludables

\begin{tabular}{|c|c|c|}
\hline Términos & $\begin{array}{l}\text { Relaciones } \\
\text { semánticas }\end{array}$ & Dominio - 2 \\
\hline $\begin{array}{l}\text { Arroz de espagueti } \\
\text { Pollo, lenteja, frijol rojo } \\
\text { Plátano verde cocido } \\
\text { (cabeza de gato) } \\
\text { Carne magra y pescado } \\
\text { Carne de cerdo, pavo y } \\
\text { gallina } \\
\text { Name criollo } \\
\text { Zanahoria, remolacha, } \\
\text { espinaca y habichuelas } \\
\text { Avena } \\
\text { Huevo cocido } \\
\text { Café, papa cocida, arepas } \\
\text { Extracto de guayabas y } \\
\text { níspero }\end{array}$ & Es una clase de & $\begin{array}{l}\text { Consumo de } \\
\text { dieta } \\
\text { saludable }\end{array}$ \\
\hline
\end{tabular}

Tabla 3. Alimentos que las gestantes relacionaron con el desarrollo de la hipertensión arterial

\begin{tabular}{lll}
\hline \multicolumn{1}{c}{ Términos } & $\begin{array}{l}\text { Relaciones } \\
\text { semánticas }\end{array}$ & Dominio-2 \\
\hline $\begin{array}{l}\text { Frituras en horas nocturnas } \\
\text { (salchichas, papas fritas) }\end{array}$ & $\begin{array}{l}\text { Tiene la } \\
\text { consecuencia } \\
\text { de }\end{array}$ & $\begin{array}{l}\text { Hipertensión } \\
\text { arterial en la } \\
\text { gestante } \\
\text { adolescente }\end{array}$ \\
$\begin{array}{l}\text { Alimentos con valor } \\
\text { nutricional limitado (Pizza, } \\
\text { golosinas, postres, bebidas }\end{array}$ & \\
azucaradas, perros calientes, & & \\
bizcochos, hamburguesas) & & \\
Alimentos procesados en & & \\
paquete & & \\
Sancochos con abundante & & \\
aceite & & \\
Suero y queso salado & & \\
Guineo maduro & \\
Chocolate & \\
Arroz tostado (Pegao o \\
cucayo del arroz)
\end{tabular}

\section{Discusión}

Una alimentación equilibrada, es una necesidad, un reto y algunas veces una dificultad para muchos seres humanos, al solventar dicha necesidad es donde se 
realizan actividades que se convierten en hábitos y prácticas saludables y no saludables para la vida(14). Las adolescentes con hipertensión inducida por la gestación, no son la excepción, la cultura donde se desenvuelven es diversa. Los alimentos ingeridos pueden contribuir a su bienestar o a la enfermedad.

Una de las participantes cataloga el plátano como alimento saludable: Participante 1: "El plátano verde cocido y machacado (cabeza de gato) alimenta y es saludable". Lo anteriormente expresado, coincide con lo planteado por la Organización Mundial de la Salud(15), que considera la hipertensión, un factor de riesgo para las enfermedades cardiovasculares, como los infartos de miocardio y los eventos cerebrovasculares, donde el consumo de alimentos ricos en potasio como el plátano, banano, y la espinaca reducen la presión arterial. De igual forma, algunos autores sostienen que el plátano es un alimento con múltiples beneficios para la salud(16).

Dentro de los alimentos saludables también se encuentra el pescado, asociado con menor riesgo de desarrollo de enfermedad coronaria(17,18). Según un estudio realizado en Medellín (Colombia), resaltó que el consumo de pescado tiene grandes beneficios, entre ellos: prevención de las enfermedades cardiovasculares, de Alzheimer, disminuye el riesgo de padecer diabetes; además reporta en nuestro país el consumo frecuente de bocachico, bagre, trucha y tilapia, provenientes del mar y de algunos ríos(19).

Los tubérculos como el ñame criollo, se destacan como alimentos que garantizan el bienestar en la gestación(20,21), así lo expresó la madre de la participante 9: "El ñame es beneficioso porque nutre a la mujer que lleva un bebé en su vientre.

El ñame es un tubérculo propio de la costa caribe colombiana, donde se encuentran cinco variedades de ñame: criollo, espino, ñame papa, ñame azúcar y ñampí, pero el de mayor consumo es el ñame criollo siendo la principal fuente de empleo rural y oferta de alimento para los habitantes(20). Este tubérculo contiene vitaminas $\mathrm{C}, \mathrm{A}$ y $\mathrm{B}$, antioxidantes, fibra y potasio, indispensable para regular la frecuencia cardiaca y la presión arterial(21), validando así lo expresado por la madre de la gestante.

El consumo de pan fue considerado por las participantes como un alimento relacionado con el desarrollo de la hipertensión, como lo expresaron en las entrevistas, la participante 2: "No comía comida chatarra pa' decir, yo digo que fue el pan que me engordó y me subió la presión, lo comía dos o tres veces al día, panes de queso o de arequipe", esto se relaciona con lo expresado por la participante 5: "Me gustaba mucho el pan, cada ratico comía panes de mantequilla, de queso, yo pienso que me subieron la presión, porque tenían bastante harina"; cuando se consumen rellenos de dulces el peligro es mayor, relacionándose con los referentes de la Organización Mundial de la Salud(22,23), al señalar los panes como productos muy elaborados, con alto contenido de sal, lo que se asocia a un mayor riesgo de desarrollo de hipertensión, cardiopatías y eventos cerebrovasculares.

Respecto a las frituras, estas hacen parte de los alimentos que causan morbilidad(24,25), y así lo manifiesta la Participante 3: "Las frituras tienen abundante grasa mala para la presión". El alto consumo de frituras se convierte en un factor de riesgo para la salud cardiovascular, principalmente por su alta densidad energética que se presenta a expensas de la grasa y por la formación de compuestos tóxicos, relacionados con el desarrollo de algunos tipos de cáncer(24). Es necesario resaltar que, al freír, el aceite y los alimentos sufren cambios estructurales y bioquímicos, por lo tanto, se modifican las propiedades del producto final(24).

El arroz tostado, denominado en la cultura de la Costa Caribe de Colombia como "pegao o cucayo", fue señalado por una participante como un alimento relacionado con la hipertensión: "El cucayo aumenta la presión y al comerlo hace que mi matrizy la placenta se peguen" (Entrevista realizada a la participante 7). En relación a esto Mendoza L. et al.(26), afirman que la hipertensión arterial, es una de las principales causas del desprendimiento placentario, complicación que se relaciona con la morbimortalidad materno fetal; por lo tanto, si bien el consumo de cucayo no conlleva directamente al acretismo placentario, su consumo puede convertirse en un factor de riesgo para la hipertensión y esta enfermedad puede originar el desprendimiento de la placenta(27).

Los resultados del presente estudio analizados desde la perspectiva de los cuidados enfermeros propuestos por la teorista Madeleine Leininger, conllevan a establecer que las prácticas de cuidado cultural que se deben mantener en las participantes, son: ingesta de frutas (mandarinas, granadillas, guayabas, nísperos), hortalizas (remolachas, zanahorias, entre otras), proteínas (pollo, carnes magras, pescados, entre otras), tubérculos (ñame criollo, papa cocida), plátano en "cabeza de gato" y leguminosas (lentejas, frijol rojo). Mientras que las prácticas a negociar con la adolescente gestante son: la ingesta de guineo maduro y chocolate, su consumo debe ser de forma 
moderada, para que no resulte perjudicial en la gestación.

Se deben evitar el consumo de frituras, alimentos procesados en paquete $\mathrm{y}$ aquellos con valor nutricional limitado que aportan bajo contenido de proteínas, vitaminas, minerales y alto contenido de azúcar, grasa y sal.

Finalmente, es indispensable conocer el significado de estas prácticas de cuidado, con el propósito de fortalecer la salud materno-perinatal en la vida actual y futura; los resultados del estudio constituyen la base para la implementación de proyectos direccionados a promover los cuidados culturales humanizados en los servicios de atención prenatal que ofrecen las instituciones prestadoras de servicios de salud de la región. Además, puede contribuir en la planeación de estrategias desde las secretarías de salud municipal y departamental, y en la actualización de currículos en las facultades de ciencias de la salud de las instituciones de educación superior.

\section{Conclusiones}

La alimentación en las adolescentes con hipertensión inducida por la gestación, se relaciona con las creencias y costumbres de la región caribe, en la que se evidencia la diversidad de significados en las prácticas cotidianas inmersas en el entorno de cada adolescente, en las subcategorías surgidas, tales como, alimentos saludables y aquellos relacionados con la hipertensión arterial.

El conocimiento del significado de las prácticas de cuidados en la alimentación de las gestantes hipertensas conlleva a retomar el liderazgo y empoderamiento del personal de enfermería en la interacción diaria con las gestantes adolescentes, centrando los cuidados en el entorno cultural acorde a las necesidades reales, y no únicamente al entorno hospitalario, lo que contribuirá a brindar cuidados de enfermería coherentes y humanizados, que generen satisfacción y bienestar en el sujeto de atención. De igual forma permite al profesional de enfermería implementar programas y/o proyectos de intervención a nivel domiciliario e institucional en este grupo poblacional.

\section{Recomendaciones}

Es pertinente el desarrollo de investigaciones donde se intervenga a esta población vulnerable, sin olvidar la normatividad nacional como, la Resolución 3280 de 2018, referenciada en el Análisis de Salud en Latinoamérica, aspecto fundamental en el marco legal de salud en Colombia para la mejora continua en el sistema y específicamente en la población maternoperinatal.

Conflicto de intereses: Ninguno declarado por los autores.

\section{Referencias}

1. García Odio AA, González Suárez M. Factores de riesgo asociados a embarazadas adolescentes. Rev Ciencias Médicas. 2018;22(3):416-27. Disponible en: http://scielo.sld.cu/pdf/rpr/v22n3/rpr02318.pdf

2. Córdoba Solano AM, Niño Estupiñan IP. Factores de riesgo obstétrico, clínico y puerperio en el embarazo en mujeres en edad fértil [Tesis de grado]. Bucaramanga: Universidad Cooperativa de Colombia; 2019. Disponible en: https://repository.ucc.edu.co/handle/20.500.12494/13568 ?locale $=\mathrm{es}$

3. Molina Gómez AM, Pena Olivera RA, Díaz Amores CE, Antón Soto M. Condicionantes y consecuencias sociales del embarazo en la adolescencia. Rev Cubana Obstet Ginecol. 2019;45(2):e218. Disponible en: http://scielo.sld.cu/scielo.php?script=sci_arttext\&pid=S013 8-600X2019000200017

4. OMS. El embarazo en la adolescencia. 2020 [consultado 2021 Enero 18]. Disponible en: https://www.who.int/es/newsroom/fact-sheets/detail/adolescent-pregnancy

5. Instituto Nacional de Salud de Colombia. Boletín Epidemiológico Semanal (BES): Mortalidad Materna. 2021. Disponible en: https://www.ins.gov.co/buscadoreventos/BoletinEpidemiologico/2021_Boletin_epidemiologi co_semana_21.pdf

6. Secretaria Departamental de Salud de Sucre, Colombia. Embarazo en adolescentes. Programa de Salud Sexual y Reproductiva. 2016.

7. Oficina de Estadísticas Clínica Santa María S.A.S, Colombia. Gestantes Adolescentes y Trastornos Hipertensivos del Embarazo. Referente Morbilidad Materna Extremaepidemiología 2016 -2017. 2017.

8. Sánchez-Ojeda MA, Segura-Robles A, Gallardo-Vigil MÁ, Alemany-Arrebola I. Enfermería Transcultural. Formación de los futuros profesionales de Enfermería en España. Index Enferm. 2018;27(4):247-250. Disponible en: https://scielo.isciii.es/scielo.php?script=sci_arttext\&pid=S1 132-12962018000300015

9. Escobar Castellanos B, Paravic-Klijn T. La transculturalidad, elemento esencial para mejorar la atención en salud y de enfermería. Enfermería actual en Costa Rica. 2017;(33):1-15. DOI: 10.15517/revenf.v0i33.29627.

10. Valencia Arroyave MC, Vega Cuellar J, Zapata Celín T. Universalidad y diversidad cultural, una mirada desde el cuidado de enfermería [Tesis de grado]. Santiago de Cali: Universidad Santiago de Cali; 2020. Disponible en: https://repository.usc.edu.co/handle/20.500.12421/3702

11. Garrido N. El método de James Spradley en la investigación cualitativa. Enfermería: Cuidados Humanizados. 2017;6(Especial):37-42.

DOI: 10.22235/ech.v6iespecial.1449.

12. Mateus JC, Varela MT, Caicedo DM, Arias NL, Jarmillo CD, Morales LC, et al. ¿Responde la Resolución 8430 de 1993 a las necesidades actuales de la ética de la investigación en salud con seres humanos en Colombia? Biomed. 2019;39(3):448-63. DOI: 10.7705/biomedica.4333. 
13. Congreso de Colombia. Ley 911 de 2004: Por la cual se dictan disposiciones en materia de responsabilidad deontológica para el ejercicio de la profesión de Enfermería en Colombia; se establece el régimen disciplinario correspondiente y se dictan otras disposiciones. 2004 [actualizado 2020 Diciembre 31, consultado 2021 Enero 19]. Disponible en: http://www.secretariasenado.gov.co/senado/basedoc/ley_ 0911_2004.html

14. Sandoval KV, Nieves ER, Luna MÁ. Efecto de una dieta personalizada en mujeres embarazadas con sobrepeso $\mathrm{u}$ obesidad. Revista chilena de nutrición. 2016;43(3):233-46. DOI: $10.4067 /$ S0717-75182016000300002.

15. Organización Mundial de la Salud (OMS). Aumentar la ingesta de potasio para reducir la tensión arterial y el riesgo de enfermedades cardiovasculares en adultos. 2019 [consultado 2021 Enero 21]. Disponible en: https://www.who.int/elena/titles/potassium_cvd_adults/e $\mathrm{s} /$

16. Dussán-Sarria S, Gaona-Acevedo AF, Hleap-Zapata JI. Efecto del Uso de Antioxidantes en Plátano Verde Dominico-Hartón (Musa AAB Simmonds) Cortado en Rodajas. Información Tecnológica. 2017;28(4):3-10. DOI: 10.4067/S071807642017000400002.

17. Calañas-Continente AJ. Alimentación saludable basada en la evidencia. Endocrinología y Nutrición. 2005;52(Suppl 1):824. DOI: $10.1016 /$ S1575-0922(05)74649-0.

18. Saavedra A. El Pescado, Alimento Rico y Nutritivo Que No Es Solo Para Semana Santa [Internet]. El Tiempo; 2018. Disponible

en: https://www.eltiempo.com/salud/beneficios-del-consumode-las-variedades-pescado-198060

19. Restrepo-Betancurt LF, Rodríguez-Espinosa H, Valencia-Y D. Caracterización del consumo de pescado y mariscos en población universitaria de la ciudad de Medellín - Colombia. Universidad y Salud. 2016;18(2):257-65. DOI: $10.22267 /$ rus.161802.36.

20. Salcedo-Mendoza J, García-Mogollón C, Salcedo-Hernández D. Propiedades funcionales de almidones de ñame
(Dioscorea alata). Biotecnología en el Sector Agropecuario y Agroindustrial. 2018;16(2):99-107. Disponible en: http://www.scielo.org.co/scielo.php?script=sci_abstract\&pi $\mathrm{d}=\mathrm{S} 1692-35612018000200099$ \&lng=es\&nrm=iso

21. Ñame. ABC. 2019 [consultado 2021 Enero 19]. Disponible en: https://www.abc.es/bienestar/alimentacion/abci-name201909240905_noticia.html

22. Reducir el consumo de sal. Organización Mundial de la Salud (OMS); 2020 [consultado 2021 Enero 21]. Disponible en: https://www.who.int/es/news-room/factsheets/detail/salt-reduction

23. Alimentación sana. Organización Mundial de la Salud (OMS); 2018. Disponible en: https://www.who.int/es/newsroom/fact-sheets/detail/healthy-diet

24. Montes N, Millar I, Provoste R, Martínez N, Fernández D, Morales G, et al. Absorción de aceite en alimentos fritos. Rev. chil. nutr. 2016;43(1):87-91. DOI: 10.4067/S071775182016000100013.

25. Nutrición: ¿Qué efectos tienen las frituras en el cuerpo y cómo podemos hacerlas menos dañinas? Misiones Online; 2018 [consultado 2021 Enero 20]. Disponible en: https://misionesonline.net/2018/02/10/nutricion-efectostienen-las-frituras-cuerpo-podemos-hacerlas-menosdaninas/

26. Mendoza L, Nazco A, Méndez Y, Vásquez D, Coloma $M$, Alvarez de la Rosa M. Evolución y aspectos ecográficos del desprendimiento crónico de placenta. Rev. chil. obstet. ginecol. 2017;82(3):252-8. DOI: 10.4067/s071775262017000300252 .

27. Liñayo-Chouza J, Álvarez-Silvares E, Domínguez-Vigo $P$, Alves-Pérez MT, Castro-Vilar L. Morbilidad y mortalidad a largo plazo en mujeres con antecedente de desprendimiento prematuro de placenta normoinserta. Ginecol Obstet Mex. 2019 [consultado 2021 Enero 30];87(3):167-76. Disponible en: https://www.medigraphic.com/pdfs/ginobsmex/gom2019/gom193c.pdf 